\title{
Vom Unbehagen in der Gesundheitsförderung
}

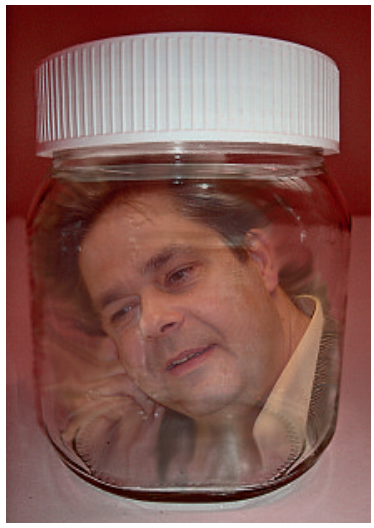

Eberhard Wolff

1 Klotter C. Warum wir es schaffen, nicht gesund zu bleiben. Eine Streitschrift zur Gesundheitsförderung. München/Basel: Ernst Reinhardt-Verlag; 2009.

2 Campos, P. The Obesity Myth: Why America's Obsession with Weight is Hazardous to Your Health. New York: Gotham; 2004.

3 «Abspeckprogramme sind Psychoterror». Ein Gespräch mit dem Ernährungsmediziner Gunter Frank. Ars Medici. 2010;2:54-7.

4 Schorb F. Dick, doof und arm? Die grosse Lüge vom Übergewicht und wer von ihr profitiert. München: Droemer; 2009.

5 Als aktueller Versuch der Verbindung von gesunder Ernährung und Genuss siehe: Schönberger G, Krekel S. Dicke sterben. Dünne auch. Vom Verdruss zum Genuss. Neustadt/W.: Umschau; 2009.

* PD Dr. rer. soc. Eberhard Wolff ist Kulturwissenschaftler, Medizinhistoriker und Mitglied der Redaktion Medizingeschichte der Schweizerischen Ärztezeitung.
Ich bekenne: Wir geben unseren Kindern Schokolade! Jeden Abend! Auch viel Schokolade, wenn sie das wollen! Und Cola! Sogar Chips! Uff, jetzt ist es draussen!

Es gibt einen Grund für dieses Coming-out. Er ist 163 Seiten stark und im letzten Jahr erschienen: eine «Streitschrift zur Gesundheitsförderung» des deutschen Ernährungspsychologen und GesundheitsförderungsFachmanns Christoph Klotter [1]. Nein es ist keine dieser Wortmeldungen, welche die Debatte um Übergewicht und falsche Ernährung grundsätzlich als Mythos [2], Panikmache [3] oder gar Lüge [4] bezeichnen. Lassen wir deshalb die Frage beiseite, wie weit Schokolade, Cola und Chips wirklich ungesund sind. Das Buch liefert andere Erklärungen, warum wir unseren Kindern Schokolade geben und damit zu der überwältigenden gesellschaftlichen Mehrheit zählen, für die millionenschwere Programme zur Gesundheitsprävention und Ernährungsberatung aufgelegt werden, deren Erfolg letztlich doch bezweifelt wird. Es geht um die Effizienz und Legitimität der heute verbreiteten Form von Gesundheitsförderung, um ihr Scheitern und dessen Gründe. So auch der Titel: «Warum wir es schaffen, nicht gesund zu bleiben».

In der Tagesbetreuung wird unseren Kindern mit den besten Absichten «gesundes Essen» vorgesetzt, ohne dass ihnen viele Wahlmöglichkeiten bleiben. Ähnlich sieht Klotter die gesamte Gesundheitsförderung verfasst. Sie fragt die Menschen nicht, was sie wollen, sondern geht davon aus, zu wissen, was gut für sie ist. Klotter nennt das ein Partizipationsdefizit, und das sei ein Hauptmangel der Gesundheitsförderung. Er erklärt dies mit dem Menschenbild der Gesundheitsförderung: Sie sei eine der letzten verbliebenen grossen gesellschaftlichen Utopien, und - wie andere sich avantgardistisch verstehende Bewegungen auch - habe sie den Glauben, absolute Wahrheiten zu vertreten: Wer für Gesundheit eintritt, steht auf der Seite der Guten. Gesundheitsförderung ähnele damit einer «Erweckungsbewegung», sei implizit totalitär und stehe im Geiste des aufgeklärten Absolutismus, weil für sie die Selbstbestimmung des Menschen nur so lange gelte, wie er sich gesundheitsbewusst verhalte.

Gesundheitsförderung, so Klotter, vertrete aber keine letzten Wahrheiten, sondern jene spezifische Moral, die der Soziologe Max Weber als «Protestantische Ethik» bezeichnet hat. Vereinfacht gesagt: Das Leben sei nicht dazu da, zu geniessen, sondern hart zu arbeiten und produktiv zu sein. Er nannte das «innerweltliche Askese». Die Gesundheitsförderung verkaufe die Protestantische Ethik als universelle Vernunft und versuche, diese Lebensweise global vorzuschreiben.
Für die Betreuerinnen unserer Kinder ist «gesundes Essen» ein sehr wichtiges Ziel. Für unsere Kinder ist «gesundes Essen» nicht das höchste Ziel. Essen soll für sie gut schmecken und nicht nach Essenspyramiden-Ethik. Nach Klotter hat die Gesundheitsförderung die Tendenz, Gesundheit als Wert zu verabsolutieren. Spass oder Entspannung sind nur wichtig, wenn sie der Gesundheit dienen. Der Mensch hat aber bisweilen andere Bedürfnisse, als gesund zu leben: faul sein, einem «Laster» frönen, «sündigen», einen Rausch haben, ein Risiko eingehen. Der französische Denker Georges Bataille nenne dies das Grundbedürfnis der unproduktiven Verausgabung. Und warum soll «Gesundheit» immer wichtiger als «Kultur» sein?

Unsere Kinder haben sich über Jahre nicht mit dem verordneten «gesunden Essen» in ihrer Tagesbetreuung abfinden können. Nach Klotter könnte es daran liegen, dass die Empfehlungen gesunder Ernährung zu sehr mit konkreter Verhaltenskontrolle verlinkt sind. Selbst der wohlmeinende Ratschlag beinhaltet ein Stück «Disziplinarmacht» und Kontrolle über den individuellen Körper, wie sie Foucault bereits anhand der frühneuzeitlichen Gefängnisse und Spitäler beschrieben hat. Das merken auch unsere Kinder. Die Folge sind Skepsis und Verweigerung, Trotz gegen einen verordneten Lebensstil, vielleicht auch die Schokolade zu Hause am Abend. Grüne Lebensmittel nennen sie «Kindergift». Sarkasmus kann ein Ventil sein. «Rote» Rüebli und Tomaten essen sie im Übrigen freiwillig und begeistert, weil sie ihnen schmecken [5].

Ein moralgetränktes «Gut-Böse-Schema» der Gesundheitsförderung schafft nach Klotter schliesslich auch «Schuldige». Das Normale wird das Gute. Wer nicht normal ist, steht in der Gefahr, Aussenseiter zu werden. Unter den Primarschulkollegen unserer Kinder wird regelmässig darüber geredet, wer «dick» ist.

Eine zu penetrante Gesundheitsförderung kann nach Klotter mithelfen, eine Gesellschaft zu spalten in Gesundheitswillige und nicht Gesundheitswillige. Jeder Teil grenzt sich gegen den anderen ab. Pierre Bourdieu hat dies als «Distinktion» bezeichnet. Die einen leben gesund, um zu zeigen, dass sie nicht $\mathrm{zu}$ den «Ungesunden» gehören. Die «Ungesunden» grenzen sich ab, weil sie nicht zu den Besserwissern gehören wollen.

Klotter hat eine sehr pointierte, oft auch theoretische Streitschrift vorgelegt. Ein aufmerksamer Blick zeigt, dass sich vieles davon in unserem Alltag spiegelt. Nicht zuletzt entdecke ich in mir selber immer wieder ein fettes Stück Protestantischer Ethik.

Eberhard Wolf* 